\title{
A STRUCTURAL BRIDGE BETWEEN ALTERNANT AND NON-ALTERNANT HYDROCARBONS
}

Richard Francis Langler

Department of Chemistry, Mount Allison University, Sackville, N.B., Canada E4L 1G8

Recebido em 9/5/00; aceito em 29/6/00

A simple set of trimethylene-substituted even, fully- $\pi$-bonded, non-alternant monocycles is shown to have several key features in common with acyclic, even alternant polyenes at the Hückel level. These non-alternant molecules provide a bridge between alternant and non-alternant hydrocarbons. This topic might serve as a useful addition to Hückel theory courses targeted at senior undergraduate students.

Keywords: Chemical graph theory, Hückel theory, non-alternant hydrocarbon, alternant hydrocarbon.

\section{INTRODUCTION}

The longest-known and most-widely disseminated approach to molecular orbital calculations on planar hydrocarbons is due to Hückel ${ }^{1}$. One of the most important distinctions made at the Hückel level is that between alternant (e.g. 1) and non-alternant hydrocarbons (e.g. 2).

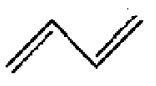

1

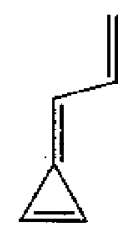

2
Non-alternant hydrocarbons invariably feature at least one ring constructed with an odd number of carbon atoms, whereas alternant hydrocarbons have no such rings ${ }^{2-4}$.

To begin to obtain the Hückel description for a hydrocarbon with $\mathrm{n}$ carbon atoms, in the traditional manner, one must (i) produce a set of $\mathrm{n}$ homogeneous linear equations, (ii) generate an $n \times n$ secular determinant and (iii) diagonalize the determinant to obtain an nth order polynomial. It is this polynomial which determines the molecular orbitals (eigenfunctions) and their energies (eigenvalues) at the Hückel level. The polynomials for alternant and non-alternant hydrocarbons are distinctly different.

From the Pairing Theorem ${ }^{5}$, it can be shown that the polynomial roots for an even alternant hydrocarbon occur in pairs whose absolute values are the same but whose signs are opposed. Hence, as observed earlier ${ }^{6}$, the polynomial for an even alternant hydrocarbon must have the form shown in equation (1).

$\prod_{i=1}^{n / 2}\left(x^{2}-r_{i}^{2}\right)=0$

$n$ where the number of carbon atoms in the $\pi$ system; $r_{i}$ is the ith root of the polynomial of interest.

When equation (1) is expanded, it must produce polynomials in which non-zero coefficients are exclusively associated with terms that have even exponents. As an example, equation (2) presents the polynomial for the alternant hydrocarbon butadiene $\mathbf{1}$.

$\mathrm{x}^{4}-3 \mathrm{x}+2=0$; roots: $\pm 1.618 ; \pm 0.618$
Polynomials for even, non-alternant hydrocarbons do not normally have any pairs of roots equal in magnitude but opposite in sign and do have non-zero coefficients for one or more terms that have odd exponents. Equation (3) presents the polynomial for the non-alternant hydrocarbon 2 .

$\mathrm{x}^{6}-6 \mathrm{x}^{4}+2 \mathrm{x}^{3}+8 \mathrm{x}^{2}-4 \mathrm{x}-1=0$

roots: $-2.228 ;-1.360 ;-0,186 ; 1 ; 1 ; 1.775$

Typically, when students are introduced to Hückel theory, alternant and non-alternant hydrocarbons are presented as distinct structural types. To the best of my knowledge, no intermediate or bridging structures have been previously described at the Hückel level. I began to seek such bridging structures in the hope that discussions of them in theoretical organic chemistry lectures would provide a smooth transition from one type of molecule to the other.

Amongst the well-known Hückel-level differences between alternant and non-alternant hydrocarbons are (i) the aforementioned root pairs characteristic of even alternant hydrocarbon polynomials and (ii) the pronounced topological polarization characteristic of non-alternant hydrocarbons. These properties will be employed to guide the search for bridging structures.

\section{RESULTS AND DISCUSSION}

The Pairing Theorem ${ }^{5}$ rules out any possibilty of Hückellevel even, alternant, fully- $\pi$-bonded hydrocarbons which do not have polynomial root pairs that are equal in magnitude and opposite in sign. Furthermore, it requires that every carbon in an even, fully- $\pi$-bonded alternant hydrocarbon be exactly neutral. Hence, alternant hydrocarbons of that type cannot have polarized $\pi$-systems at the Hückel level. The search for bridging structures must focus on non-alternant hydrocarbons which might mimic alternant structures.

Given the sample polynomials in equations (2) and (3), it would seem sensible to seek non-alternant hydrocarbons which have polynomials as similar to those for alternant hydrocarbons as possible i.e. the number of terms with odd exponents should be minimized.

With a graph-theoretical analysis, using established principles $^{7,8}$, it can be shown that there will be only one oddexponent term in the polynomial for any fully- $\pi$-bonded, even, non-alternant monocycle in which all sigma bonds involve at least one ring carbon. (This condition permits only one valid cyclic Sachs' graph for each compound). The simplest possible example, compound 3 , and its polynomial are shown in Figure 1. 


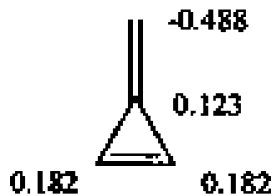

3

\section{$x^{4}-4 x^{2}+2 x+j=0$}

Figure 1.

Even, fully- $\pi$-bonded, alternant hydrocarbons do not have polarized $\pi$-systems at the Hückel level. Small molecules like 3 (non-alternant monocycle with a single exocyclic methylene) have substantial Hückel polarization (see Figure 1). I propose to use the sum of the partial positive charges $\left(\mathrm{q}_{+}\right)$to measure Hückel framework polarization for even non-alternant hydrocarbons.

Even, alternant hydrocarbon polynomials have root pairs $\pm \mathrm{r}_{\mathrm{i}}$. If the individual pairs are summed, each sum would be zero. Hence, the average of these sums would also be zero. The average of the absolute values of the corresponding polynomial root pairs for $\mathbf{3}$ is not close to zero. Table 1 presents averaged polynomial root pair sums $(\overline{\Delta E})$ and the sum of $q+(\Sigma q+)$ for a selection of non-alternant monocycles 4 .

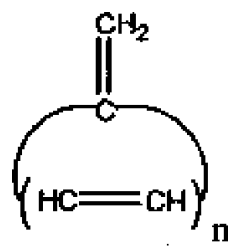

4

Table 1. Hückel calculated $\overline{\Delta \mathrm{E}^{\mathrm{a}}}$ and $\Sigma \mathrm{q}_{+}{ }^{\mathrm{b}}$ for non-alternant monocycles 4 .

\begin{tabular}{cccc}
\hline $\mathrm{n}$ & Ring Size & $\overline{\Delta \mathrm{E}}$ & $\Sigma \mathrm{q}_{+}$ \\
\hline 1 & 3 & 0.689 & 0.488 \\
2 & 5 & 0.412 & 0.378 \\
3 & 7 & 0.294 & 0.310 \\
4 & 9 & 0.229 & 0.265 \\
5 & 11 & 0.188 & 0.232 \\
6 & 13 & 0.159 & 0.206 \\
7 & 15 & 0.138 & 0.186 \\
8 & 17 & 0.122 & 0.169 \\
9 & 19 & 0.110 & 0.156 \\
10 & 21 & 0.100 & 0.143 \\
11 & 23 & 0.091 & 0.134 \\
12 & 25 & 0.084 & 0.125
\end{tabular}

$\overline{\mathrm{a}} \overline{\mathrm{E}}$ : average of absolute values of sum of polynomial root pairs $\mathrm{r}_{1}, \mathrm{r}_{\mathrm{n}} ; \mathrm{r}_{2}, \mathrm{r}_{\mathrm{n}-1} ; \mathrm{r}_{3}, \mathrm{r}_{\mathrm{n}-2} ; \ldots . . ;{ }^{\mathrm{b}} \mathrm{Sq}_{+}$: sum of partial positive charges for the structure

Inspection of the Table 1 data suggests that molecules $\mathbf{4}$, for which $\mathrm{n}$ is very large, will have $\overline{\Delta \mathrm{E}}$ and $\Sigma \mathrm{q}_{+}$values very near the alternant ideal value of zero. However, the persistence of significant magnitudes for $\Sigma \mathrm{q}_{+}$, even in relatively large nonalternant monocycles has prompted an examination of nonalternant monocyclic relatives of $\mathbf{4}$.

In an earlier study ${ }^{9}$, we have described the application of perturbation theory to the problem of anticipating topologically-induced polarity in even, fully- $\pi$-bonded, nonalternant monocycles.
It was shown there, that such non-alternant monocycles largely lose their Hückel-level polarization if more than one odd substituent is attached to the ring.

In accord with earlier discussion herein, structural modification involved the introduction of two additional methylene groups (see structure 5). Examination of a series of compounds 5 furnished the data in Table 2.

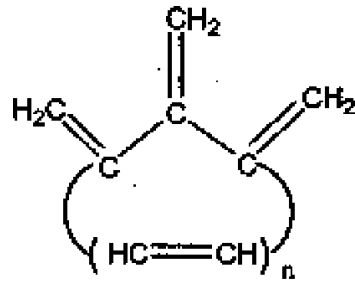

$\mathbf{5}$

Table 2. Hückel calculated $\overline{\Delta \mathrm{E}}{ }^{\mathrm{a}}$ and $\Sigma_{\mathrm{q}+}{ }^{\mathrm{b}}$ for non-alternant monocycles $\mathbf{5}$.

\begin{tabular}{cccc}
\hline $\mathrm{n}$ & Ring Size & $\overline{\Delta \mathrm{E}}$ & $\Sigma \mathrm{q}_{+}$ \\
\hline 0 & 3 & 0.667 & 0.187 \\
1 & 5 & 0.319 & 0.099 \\
2 & 7 & 0.238 & 0.059 \\
3 & 9 & 0.193 & 0.039 \\
4 & 11 & 0.167 & 0.026 \\
5 & 13 & 0.153 & 0.018 \\
6 & 15 & 0.130 & 0.014 \\
7 & 17 & 0.116 & 0.011 \\
8 & 19 & 0.105 & 0.008 \\
9 & 21 & 0.097 & 0.007 \\
10 & 23 & 0.092 & 0.005 \\
11 & 25 & 0.083 & 0.004
\end{tabular}

$\overline{\mathrm{aE}}$ : average of absolute values of sum of polynomial root

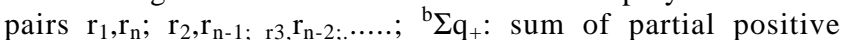
charges for the structure.

A comparison of the data in Tables 1 and 2 shows that structures $\mathbf{5}$ are slightly superior to structures $\mathbf{4}$ with regard to the average of the polynomial root sums. Structures $\mathbf{5}$ are much less polar than structures $\mathbf{4}$ as measured by Hückel $\Sigma q_{+}$values. Hence, structures $\mathbf{5}$ constitute a set of monocycles which are better mimics of alternant hydrocarbons. Algebraically, each polynomial for structures $\mathbf{4}$ and $\mathbf{5}$ has a single term with an odd exponent whose coefficient is always +2 . As the monocycles become larger and larger, the coefficients for even exponent terms in the polynomial become larger and larger e.g. the $\mathrm{x}^{12}$ coefficient for $5(\mathrm{n}=10)$ is 54,028 . The impact of the odd exponent term on the description produced from the polynomial becomes smaller and smaller so that the description for larger monocycles matches that for an alternant structure more precisely.

An ordered set of alternant-hydrocarbon-like non-alternant molecules can now be constructed beginning with structures 5 ( $\mathrm{n}$ is large) which will have very small $\overline{\Delta \mathrm{E}}$ values and no Hückel-level polarity. As the structures shrink, polynomial root pair differences will increase and the framework will become more and more strongly polarized. The alternant - non-alternant bridge might be completed by adding on the set of structures $4(\mathrm{n}=7 \rightarrow \mathrm{n}=1)$ which begins with framework polarization equal to $5(n=0)$ and increases it further.

Insight into the chemistry associated with a given structure is accessible from the frontier molecular orbitals of that structure ${ }^{10,11}$. Commonly, students are required to memorize the nodal patterns of the frontier orbitals of a few familiar 
acyclic polyenes - ethylene, butadiene and hexatriene. Butadiene and ethylene frontier molecular orbitals are particularly useful for introductory discussions of Diels-Alder reactions. Earlier ${ }^{9}$, we have enunciated a very simple method for deducing the HOMO and LUMO nodal properties for any even, acyclic polyene. The HOMO is a linear combination of ethylene HOMO's, embedded on the polyene framework so that only antibonding interactions occur between ethylene units. The LUMO is a linear combination of ethylene LUMO's, embedded on the polyene framework so that only bonding interactions occur between ethylene units. Figure 2 shows this method as it applies to the branched polyene $\mathbf{6}$.

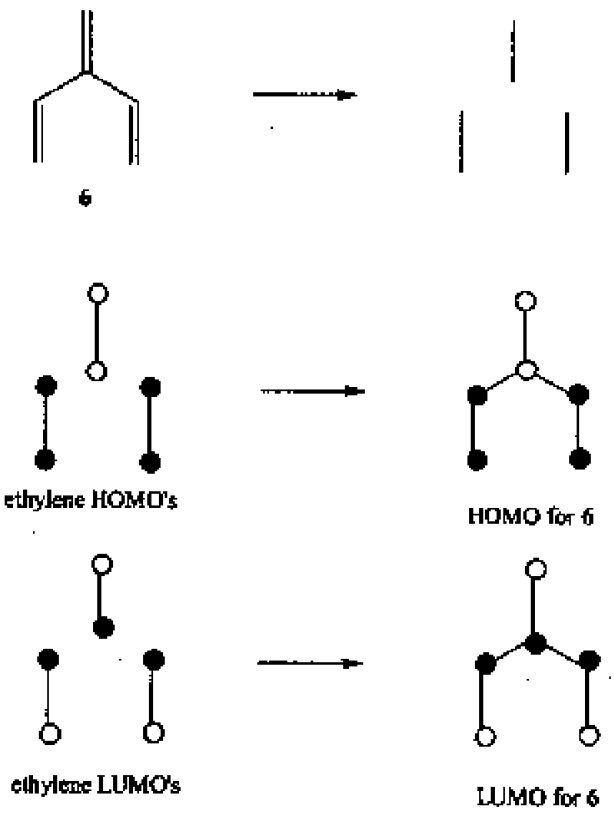

Figure 2. Construction of the frontier orbitals of 6 using ethylene frontier orbitals.

This same method works nicely to afford the nodal patterns for the frontier orbitals of structures 4 and 5 . Figure 3 presents an example of the method applied to one representative structure of each type.

Note that zero coefficients are required for an ethylene unit which cannot be positioned in the HOMO so as to have only antibonding interactions with its neighbors. Similarly, such a unit must have zero coefficients when it cannot be positioned in the LUMO so as to have only bonding interactions with its neighbors.

In summary, a set of non-alternant hydrocarbons $\mathbf{5}$ has been recognized. Very large members of this set have polynomial root pairs $\left(r_{1}, r_{n} ; r_{2}, r_{n-1} ; \ldots\right)$ which are very similar in absolute value.

These molecules have essentially no polarity at the Hückel level. Furthermore, the nodal patterns of their frontier orbitals can be deduced by embedding the HOMO with ethylene HOMO's and the LUMO with ethylene LUMO's. In all these ways, very large members of compounds $\mathbf{5}$ are very much like alternant, acyclic polyenes. As one proceeds through the set to smaller and smaller members, the structures $\mathbf{5}$ become more and more strongly polarized and the absolute values of their polynomial root pairs separate in magnitude (see Table 2). Ultimately, the smallest member $(\mathbf{5}, \mathrm{n}=0$, three-membered ring) is polarized to the same extent that its simpler non-alternant cousin is (4, $\mathrm{n}=7$, fifteen-membered ring).

\section{REFERENCES}

1. Hückel, E.; Z. Phys 1931, 70, 204.
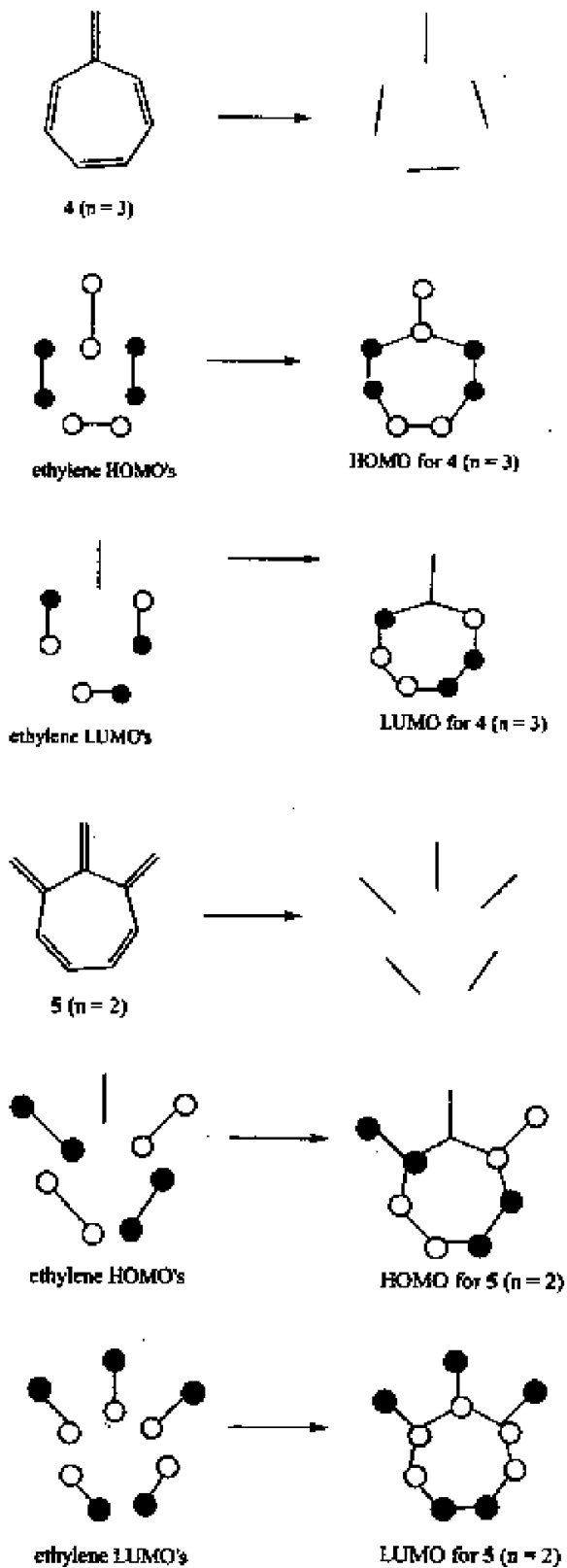

Figure 3. Construction of the frontier orbitals of $4(n=3)$ and 5 $(n=2)$ using ethylene frontier orbitals.

2. Higasi, K.; Rembaum, A.; Baba, H.; Quantum Organic Chemistry, Interscience, New York, 1965.

3. Liberles, A.; Introduction to Molecular Orbital Theory, Holt, Rinehart and Winston, New York, 1966.

4. Yates, K.; Hückel Molecular Orbital Theory, Academic Press, New York, 1978.

5. Coulson, C. A.; Rushbrook, G. S.; Proc. Cambridge Philos. Soc. 1940, 36, 193.

6. Langler, R. F.; Aust. J. Chem. 1991, 44, 297.

7. Trinajstic, N., Chemical Graph Theory, Vols. 1 and 2, CRC Press, Boca Raton, Florida, 1983.

8. Dias, J. R.; Molecular Orbital Calculations Using Chemical Graph Theory, Springer-Verlag, Heidelberg, 1993.

9. Baum. J. C.; Martin, E. D.; Ginsburg, J. L.; Langler, R. F.; Can. J. Chem. 1995, 73, 1719.

10. Fleming, I.; Frontier Orbitals and Organic Chemical Reactions, Wiley, New York, 1976.

11. Miller, B.; Advanced Organic Chemistry, Prentice Hall, Upper Saddle River, New Jersey, 1998. 